\title{
Dielectric Loading of Electric Dipole Antennas
}

\author{
Janis Galejs
}

\author{
Contribution from Applied Research Laboratory, Sylvania Electronic Systems, a Division of Sylvania \\ Electric Products Inc., 40 Sylvan Road, Waltham 54, Mass.
}

(Received March 12, 1962; revised March 29, 1962)

\begin{abstract}
It has been indicated by Wheeler that dielectric loading $(\epsilon>1)$ decreases the radiation power factor of small capacitor type antennas. A wide-angle biconical antenna is shown to follow this behavior. However, exceptions to it are provided by an infinitesimal dipole embedded in a finite dielectric sphere and by dielectrically loaded small-angle biconical antennas, where a moderate increase of $\epsilon$ provides a slight increase of the radiation power factor. In the latter two cases the dielectric surface is not tangential to the electric field lines and the reasoning which leads to the results of Wheeler is not strictly applicable.
\end{abstract}

\section{Introduction}

The limitations of small condenser-type antennas (electric dipoles) have been discussed by Wheeler [1947], who indicates that dielectric loading of the antenna increases its susceptance $B$, does not alter the radiation conductance $G$, and decreases the antenna radiation power factor $p=G / B$. This result appears to be based on heuristic reasoning and it may be of interest to examine the radiation characteristics of related dipole configurations, which are more amenable to an analysis. The specific geometries considered in this note involve an infinitesimal electric dipole which is embedded in a finite dielectric sphere and dielectrically loaded biconical antennas.

The field components of the small dipole can be obtained from a scalar solution of the wave equation [Sommerfeld, 1949]. Wait [1952] has applied this technique to a similar geometry involving a magnetic dipole. The electric dipole is discussed in section 2 and it is shown that moderate relative dielectric constants of the sphere $(1<\epsilon<4)$ provide a slight improvement of the power factor, $p$, if the boundary of the dielectric sphere is within the near zone of the dipole. This is contrary to the expectations [Wheeler, 1947].

The problem of a small-angle biconical antenna embedded in a dielectric sphere has been solved by Tai [1948]. Although numerical results are available for antennas of dimensions comparable to a wavelength [Polk, 1959], there has been no mention of the limit of small-sized antennas. The power factor of such a biconical antenna is shown in section 3.1 to exhibit approximately the same dependence on dielectric loading as the small dipole. For wideangle cones of small size the variational formulation of Tai [1949] can be readily adapted to dielectric antenna loading, as indicated in section 3.2. The dielectric loading decreases the radiation power factor in the same way as for the finite capacitor antenna of Wheeler [1947].

The $\epsilon$ dependence of the wide-angle biconical antenna and of the finite capacitor antenna may be explained by a heuristic argument, which is outlined in section 4. This argument is shown to be nonapplicable to the small-angle biconical antenna and to the infinitesimal electric dipole because the boundary of the dielectric sphere does not follow the lines of the electric field.

\section{The Short Dipole}

An electric dipole is located in the center of a dielectric sphere of radius $a$ and of dielectric constant $\epsilon_{1}$, magnetic permeability $\mu_{0}$, and conductivity $\sigma_{1}$. The outer medium is characterized by $\epsilon_{2}, \mu_{0}$, and $\sigma_{2}$. For a suppressed $e^{i \omega t}$ time variation of the fields, the field components are given by [Sommerfeld, 1949]

$$
\begin{aligned}
& E_{\theta i}=\frac{1}{r} \frac{\partial^{2}\left(r u_{i}\right)}{\partial \theta \partial r} \\
& H_{\phi i}=-\left(\sigma_{i}+i \omega \epsilon_{i}\right) \frac{\partial u_{i}}{\partial \theta} \\
& E_{r i}=-\frac{1}{r \sin \theta} \frac{\partial}{\partial \theta}\left(\sin \theta \frac{\partial u_{i}}{\partial \theta}\right) \\
& E_{\phi i}=H_{r i}=H_{\theta i}=0
\end{aligned}
$$

where $u_{i}$ satisfies the scalar wave equation

$$
\begin{gathered}
\nabla^{2} u_{i}+k_{i}{ }^{2} u_{i}=0 \\
k_{i}{ }^{2}=\omega^{2} \mu_{0} \epsilon_{i}-i \omega \mu_{0} \sigma_{i} .
\end{gathered}
$$

Solutions of (5) are given by Stratton [1941] as

$$
\begin{gathered}
f_{n}^{(1)}=A_{n} j_{n}\left(k_{i} r\right) P_{n}(\cos \theta) \\
f_{n}^{(4)}=A_{n} h_{n}^{(2)}\left(k_{i} r\right) P_{n}(\cos \theta)
\end{gathered}
$$

where $j_{n}(z)$ and $h_{n}^{(2)}(z)$ are the spherical Bessel functions of the first and fourth kind respectively and where $P_{n}(\cos \theta)$ is a Legendre polynomial of order 
$n$. The solution $f_{n}^{(1)}$ remains finite at the origin; $f_{n}^{(4)}$ represents fields whose surfaces of constant phase travel radially outward. The dipole fields in a homogeneous infinite medium may be derived from (8) after letting $n=1$. Thus, the $H_{\phi 1}$ component of the dipole of moment $I d s$ is given by

$$
H_{\phi 1}=\frac{I d s}{4 \pi r^{2}}\left(1+i k_{1} r\right) \sin \theta e^{-i k_{1} r} .
$$

It is also given by (2) and (8) as

$$
H_{\phi 1}=-\left(\sigma_{1}+i \omega \epsilon_{1}\right) A \frac{\partial}{\partial \theta}\left[h_{1}^{(2)}\left(k_{1} r\right) P_{1}(\cos \theta)\right]
$$

where

$$
h_{1}^{(2)}(z)=\left(\frac{i}{z^{2}}-\frac{1}{z}\right) e^{-i z}
$$

and

$$
P_{1}(\cos \theta)=\cos \theta \text {. }
$$

After carrying out the differentiation in (10) it follows that

$$
A=-\frac{\omega \mu_{0} I d s}{4 \pi} .
$$

Assuming (8) as the primary excitation, suitable solutions for the two regions are

$$
\begin{array}{rr}
u_{1}=A\left[h_{1}^{(2)}\left(k_{1} r\right)+B j_{1}\left(k_{1} r\right)\right] \cos \theta & \text { for } 0<r \leq a \\
u_{2}=C h_{1}^{(2)}\left(k_{2} r\right) \cos \theta & \text { for } a \leq r<\infty .
\end{array}
$$

Continuity of the tangential electric and magnetic field components at $r=a$ requires that $\left(\sigma_{i}+i \omega \epsilon_{i}\right) u_{i}$ and $\partial\left(r u_{i}\right) / \partial r$ are continuous. This results in

$$
\begin{gathered}
h_{1}^{(2)}(z)+B j_{1}(z)=\frac{\sigma_{2}+i \omega \epsilon_{2}}{\sigma_{1}+i \omega \epsilon_{1}} \frac{C}{A} h_{1}^{(2)}(y) \\
\frac{\partial}{\partial z}\left[z h_{1}^{(2)}(z)+B z j_{1}(z)\right]=\frac{C}{A} \frac{\partial}{\partial y}\left[y h_{1}^{(2)}(y)\right]
\end{gathered}
$$

where

$$
\begin{aligned}
& z=k_{1} a \\
& y=k_{2} a .
\end{aligned}
$$

The spherical boundary between the two regions at $r=a$ is assumed first to lie in the near fields of the dipole. For $k_{1} a, k_{2} a<<1$, small argument approximations of the spherical Bessel functions may be used in (16) and (17). Noting that

$$
\begin{gathered}
j_{1}(x) \approx \frac{x}{3} \\
h_{1}^{(2)}(x) \approx \frac{x}{3}+i\left(\frac{1}{x^{2}}+\frac{1}{2}\right)
\end{gathered}
$$

and letting $\sigma_{i}=0,(16)$ and (17) are solved for $C$ as

$$
C=A \frac{3 \epsilon_{2}}{\epsilon_{1}+2 \epsilon_{2}} .
$$

With $C$ and $A$ determined the radiation fields of the dipole are computed by substituting (15) in (1). Using the large argument approximation of the spherical Bessel function in (15)

$$
h_{1}^{(2)}(x) \approx-\frac{1}{x} \epsilon^{-i x}
$$

it follows that

$$
E_{\theta 2}=\frac{i \omega \mu_{0} I d ; \sin \theta}{4 \pi r} \frac{3 \epsilon_{2}}{\epsilon_{1}+2 \epsilon_{2}} e^{-i k_{2} r}
$$

The radiated power is computed as

$$
W=\frac{1}{2} \sqrt{\frac{\epsilon_{2}}{\mu_{0}}} \int_{0}^{2 \pi} \int_{0}^{\pi}\left|E_{\theta 2}\right|^{2} r^{2} \sin \theta d \theta d \phi .
$$

The radiation resistance follows from (25) as

$$
R=\frac{2 W}{I_{0}^{2}}=\left(\frac{3 \epsilon_{2}}{\epsilon_{1}+2 \epsilon_{2}}\right)^{2} \sqrt{\frac{\mu_{0}}{\epsilon_{2}}} \frac{k_{2}^{2}(d s)^{2}}{6 \pi} .
$$

The capacity of the dipole antenna is

$$
C=\frac{\epsilon_{1} A}{d s}
$$

where $A$ is its effective area. The radiation power factor is computed from (26) and (27) as

$$
p=\frac{R}{X}=R \omega C=\frac{9 \epsilon_{1} \epsilon_{2}}{\left(\epsilon_{1}+2 \epsilon_{2}\right)^{2}} \frac{k_{2}^{3} A d s}{6 \pi} .
$$

After designating the effective antenna volume $A d s$ by $V$ and letting $\epsilon=\epsilon_{1} / \epsilon_{2}$ (28) becomes

$$
p=\frac{9 \epsilon}{(\epsilon+2)^{2}} \frac{k_{2}^{3} V}{6 \pi} .
$$

The radiation power factor $p$ of (29) is increased slightly for $1<\epsilon<4$, but it decreases for larger values of $\epsilon$.

If the spherical boundary between the two regions at $r=a$ lies in the far fields of the dipole, $k_{1} a$ and $k_{2} a$ $>>1$ and large argument approximations of the spherical Bessel functions may be used in (16) and (17). Noting that

$$
j_{1}(x) \approx-\frac{1}{x} \cos x
$$

and applying (23), eqs (16) and (17) may be solved for $C$ as in

$$
C=A e^{i y} \frac{\sqrt{\epsilon_{1}}}{\sqrt{\epsilon_{1}} \cos z+i \sqrt{\epsilon_{2}} \sin z} .
$$

Substituting (13), (15), (23), and (31) in (1) the 
radiated electric fields become

$E_{\theta 2}=\frac{i \omega \mu_{0} I d s \sin \theta}{4 \pi r} e^{-i k_{2}(r-a)}\left[\cos k_{1} a+i \sqrt{\frac{\epsilon_{2}}{\epsilon_{1}}} \sin k_{1} a\right]^{-1}$

The radiation resistance of the dipole is computed as in (25) and (26). It follows that

$$
R=\sqrt{\frac{\mu_{0}}{\epsilon_{2}}} \frac{k_{2}^{2} d s^{2}}{6 \pi} \frac{1}{\cos ^{2} k_{1} a+\frac{\epsilon_{2}}{\epsilon_{1}} \sin ^{2} k_{1} a} .
$$

The radiation power factor $p$ is obtained from (27) and (33) as

$$
p=R \omega C=\frac{\left(\epsilon_{1} / \epsilon_{2}\right)}{\cos ^{2} k_{1} a+\frac{\epsilon_{2}}{\epsilon_{1}} \sin ^{2} k_{1} a} \frac{k_{2}^{3} A d s}{6 \pi} .
$$

The radiation power factor $p$ of (34) is increased with increasing values of $\epsilon=\epsilon_{1} / \epsilon_{2}$. This increase is quadratic with $\epsilon$, if $k_{1} a=\left(n+\frac{1}{2}\right) \pi$, and linear if $k_{1} a=n \pi$.

The fields of a dipole which is in the center of a dielectric sphere can be also derived from the Hertz vector or from the vector potential, which are parallel to the axis of the dipole. Suitable solutions for the dielectric sphere are obtained by superimposing the functions $e^{i k_{1} r} / r$ and $e^{-i k_{1} r} / r$. Outside the sphere the solution is simply $e^{-i k_{2} r} / r$. The tangential electric and magnetic fields are continuous across the boundary of the sphere at $r=a$ and one obtains $E_{\theta 2}$ of (24) or (32) depending on the radius $a$. Details of this development are shown in the appendix, section 5.2 .

\section{Biconical Antenna}

\subsection{Small-Angle Cones}

The terminal admittance of a dielectric filled small angle biconical antenna has been derived by Tai [1948] as

$$
\begin{aligned}
Y_{t}=-\frac{i \sqrt{\mu_{0} / \epsilon_{2}} \sqrt{\epsilon}}{\pi K_{2}^{2}} \sum_{k=1,3} & \frac{2 k+1}{k(k+1)} \\
& {\left[\sqrt{\epsilon} \frac{R_{k}^{\prime}\left(k_{2} a\right)}{R_{k}\left(k_{2} a\right)}-\frac{S_{k}^{\prime}\left(k_{1} a\right)}{S_{k}\left(k_{1} a\right)}\right]^{-1} }
\end{aligned}
$$

where

$$
\begin{gathered}
\epsilon=\epsilon_{1} / \epsilon_{2} \\
K_{i}=\frac{1}{\pi} \sqrt{\frac{\mu_{0}}{\epsilon_{i}}} \log \left(\cot \frac{\theta_{0}}{2}\right) \\
R_{k}(x)=\sqrt{x} H_{k+1 / 2}(x)
\end{gathered}
$$

$$
S_{k}(x)=\sqrt{ } x J_{k+1 / 2}(x) .
$$

$a$ is the length of the cone and also the radius of the dielectric boundary, $\theta_{0}$ is the apex half-angle of the cone, $H_{n}^{(2)}(x)$ is the Hankel function of the second kind of order $n$, and $J_{n}(x)$ is the Bessel function of order $n$. The prime denotes differentiation with respect to the argument of the respective function. The terminal admittance of the antenna is related to its input admittance as

$$
Y_{i}=\frac{1}{K_{1}} \frac{K_{1} Y_{t} \cos k_{1} a+i \sin k_{1} a}{\cos k_{1} a+i K_{1} Y_{t} \sin k_{1} a} .
$$

When restricting the consideration to small antennas where $k_{1} a, k_{2} a<<1$, it is permissible to use small argument approximations for the Bessel and Hankel functions of (35) and for the trigonometric functions of (40). After noting that

$$
\begin{gathered}
\frac{R_{k}^{\prime}(x)}{R_{k}(x)} \approx \begin{cases}-\frac{1}{x}\left(1+i x^{3}\right) & \text { for } k=1 \\
-\frac{k}{x} & \text { for } k \neq 1\end{cases} \\
\frac{S_{k}^{\prime}(x)}{S_{k}(x)} \approx+\frac{k+1}{x}
\end{gathered}
$$

some algebra results in

$$
\begin{aligned}
Y_{i} \approx & \frac{\sqrt{\mu_{0} / \epsilon_{2}}}{\pi K_{2}^{2}}\left(k_{2} a\right)^{4} \frac{3}{2}\left(\frac{\epsilon}{\epsilon+2}\right)^{2} \\
& +\left[\frac{\epsilon}{K_{2}}+\frac{\sqrt{\mu_{0} / \epsilon_{2}}}{\pi K_{2}^{2}} \sum_{k=1,3}, \ldots \frac{2 k+1}{k(k+1)(k \epsilon+k+1)}\right] i k_{2} a
\end{aligned}
$$

For $\epsilon=1$ the summation over $k$ is equal to $2 \log 2$, and the input admittance of (43) can be seen to be in agreement with eq VIII. 10.9 of King [1956]. The radiation power factor is computed from $Y_{i}=G+i B$ as

$$
p=\frac{G}{B} \approx \frac{3 \epsilon}{2(\epsilon+2)^{2}} \frac{\left(k_{2} a\right)^{3}}{\log \left(\cot \frac{\theta_{0}}{2}\right)}\left[1+\frac{S_{k}}{\epsilon \log \left(\cot \frac{\theta_{0}}{2}\right)}\right]^{-1}
$$

where $S_{k}$ stands for the summation over $k$ in (43). For $\theta_{0}<<1$ the term of (44) which is proportional to $S_{k}$ will be much less than unity. The power factor $p$ of (44) exhibits then essentially the same dependence on $\epsilon$ as $p$ of a small dipole in (29). For $\epsilon=1$, $p$ of (44) is equal to $p$ of a short, thin wire antenna, if the wire radius $s=a \theta_{0}$.

The cases where the inequality $k_{1} a, k_{2} a<<1$ is not satisfied have been adequately covered by Polk [1959] and will not be discussed in this note. 


\subsection{Wide-Angle Cones}

Wide-angle cones can be conveniently analyzed in terms of the variational formulation of Tai [1949]. The terminal admittance $Y_{t}$ is computed after solving an integral equation for the distribution of the electric field $E_{\theta}$ in the aperture $r=a, \theta_{0}<\theta<\left(\pi-\theta_{0}\right)$. For short, wide-angle cones the zero-order aperture field

$$
E_{\theta}=\frac{A_{0}}{\sin \theta}
$$

provides a terminal admittance $Y_{t 0}$ which differs negligibly from values of $Y_{t}$ obtained by using more accurate approximations to $E_{\theta}$.

An extension of this variational formulation to geometries where the spherical space between the cones is filled with a dielectric is straight forward, as seen from the appendix, section 5.2. The zero-order terminal admittance $Y_{t 0}$ which is computed for the aperture field of (45) is independent of the dielectric loading. It is given by Tai [1949] as

$$
Y_{i 0}=-\frac{i \sqrt{\mu_{0} / \epsilon_{2}}}{\pi K_{2}^{2}} \sum_{k=1,3, \ldots} \frac{(2 k+1) P_{k}^{2}\left(\cos \theta_{0}\right)}{k(k+1)} \frac{R_{k}\left(k_{2} a\right)}{R_{k}^{\prime}\left(k_{2} a\right)}
$$

where $P_{k}\left(\cos \theta_{0}\right)$ is a Legendre polynomial of order $k$. The difference between the first order admittance $Y_{t 1}$ and $Y_{t 0}$ of (46) is decreased in presence of dielectric loading and can be shown to approach zero for short antennas as $\theta_{0}$ approached $\frac{\pi}{2}$. The zero order aperture field of (45) is therefore sufficiently accurate also in presence of dielectric loading. $Y_{t 0}$ of $(46)$ is the same as the wide-angle admittance of Schelkunoff [1943] in his eq (11.9-9). For $k_{2} a<<1$ only the $k=1$ term of (46) gives a significant contribution to the conductance $G_{t 0}=\operatorname{Re} Y_{t 0}$. The susceptance $B_{t 0}=\mathrm{Im}$ $Y_{t 0}$ is given by a slowly converging series which becomes logarithmically infinite as $\theta_{0}$ approaches $\pi / 2$. Using the $B_{t 0}$ approximation of Schelkunoff [1943], $Y_{t 0}$ becomes

$$
\begin{aligned}
Y_{t 0}=G_{t 0}+i B_{t 0} & \approx \sqrt{\frac{\epsilon_{2}}{\mu_{0}}}\left[\frac{3 \pi}{2}\left(k_{2} a\right)^{4}\right. \\
& \left.+i 2 k_{2} a\left\{\frac{3}{2}-\log \left[4\left(\frac{\pi}{2}-\theta_{0}\right)\right]\right\}\right]
\end{aligned}
$$

as $\theta_{0}$ approaches $\frac{\pi}{2}$. For $k_{1} a<<1, Y_{i}$ of (40) is approximated by

$$
Y_{i}=G_{i}+i B \approx Y_{t}+i \sqrt{\frac{\epsilon_{2}}{\mu_{0}}} \frac{\pi \epsilon k_{2} a}{\frac{\pi}{2}-\theta_{0}} .
$$

Substituting (47) in (48) the radiation power factor $p$ is computed as

$$
\begin{aligned}
p & =\frac{G}{B}=\frac{\frac{3 \pi}{2}\left(k_{2} a\right)^{3}}{\frac{3}{2}-\log \left[4\left(\frac{\pi}{2}-\theta_{0}\right)\right]+\frac{\pi \epsilon}{\frac{\pi}{2}-\theta_{0}}} \\
& \approx \frac{3}{2 \epsilon}\left(k_{2} a\right)^{3}\left(\frac{\pi}{2}-\theta_{0}\right)
\end{aligned}
$$

as $\theta_{0}$ approaches $\frac{\pi}{2}$. The power factor of the biconical antenna of (49) may be compared with the power factor $p_{c}$ of a condenser type antenna. It follows from eqs (5) and (13) of Wheeler [1947], that dielectric loading $(\epsilon>1)$ decreases $p_{c}$ in the same way as $p$. If both antennas have equal radii and equal aperture heights, $h, h=2 a\left(\frac{\pi}{2}-\theta_{0}\right)$ and $p=4.5 p_{c}$.

The biconical antenna of wide angles $\theta_{0}$ exhibits therefore a larger power factor than the capacitor antenna of comparable size.

\section{Discussion}

The small dipole and the small-angle biconical antenna were shown to exhibit a different dependence on dielectric loading $\epsilon$ than the wide-angle biconical antenna and the capacitor antenna of Wheeler [1947].

The characteristics of the capacitor antenna and of the wide-angle biconical antenna can be deduced from simple reasoning, which may go as follows:

Dielectric is assumed to be inserted with rotational symmetry in a capacitor antenna of same symmetry. The boundary of the dielectric is made to follow the electric field lines* between the two plates of the capacitor. The electric fields in and near the capacitor and the magnetic fields outside the dielectric remain unaltered if the voltage $V$ of the capacitor is maintained constant.

The fields outside the volume occupied by the antenna and the dielectric depend on the electric and magnetic fields on the boundary surface. The radiation fields and the radiated power $P$ remain constant and do not depend on dielectric loading of the antenna as long as the fields remain constant on the fixed boundary surface. The radiation conductance $G=P / V^{2} r m s$ remains constant. However, the antenna capacity and current $I$ are increased. The antenna radiation resistance $R=P / I^{2}{ }_{r m s}$ is decreased.

The requirement that the dielectric boundary is tangential to the electric field $E$ is an essential part of the argument. This requirement is not met with the small dipole and with the small-angle biconical antenna, which exhibit sizeable radial electric field components at the dielectric boundary. This may account for the different $\epsilon$ dependence of the latter antennas.

\footnotetext{
*In the electrostatic problem of two electrodes in an infinite homogeneous dielectric the electric field $E$ does not depend on the dielectric constant $\epsilon$ as long as the potential $V$ between the electrodes is constant. The infinite homogencous dielectric may be separated into regions I and II in such a way that the closed boundary surface is tangential to $E$. If region $I$ is filled with dielectric $\epsilon_{1}$ and region II by $\epsilon_{2}$, the previous solutions for homogeneous $\epsilon_{1}$ and $\epsilon_{2}$ are applicable to their respective regions because the previous solutions satisfy all the boundary conditions including $\mathrm{E}_{1 \mathrm{t}}=\mathrm{E}_{2 \mathrm{t}}$ and $\mathrm{E}_{1 \mathrm{n}}=\mathrm{E}_{2 \mathrm{n}}=0$ on the interface between the regions. Thus, the electric field lines are not perturbed, if a dielectric boundary follows the field lines of the homogeneous dielectric.
} 
It is also possible to provide dielectric loading of a finite capacitor antenna and of wide angle biconical antennas with $E$ not tangential to the dielectric boundary. The previous reasoning and also the analysis of section 3.2 will not be strictly applicable to such antennas.

\section{Appendix}

\subsection{Derivation of the Dipole Fields in Terms of the Vector Potential}

The vector potential of the dipole is in a direction parallel to the dipole axis. The field components $E_{\theta}$ and $H_{\phi}$ can be derived from the vector potential $\mathbf{A}=1_{2} A_{2}$ as

$$
\begin{gathered}
E_{\theta}=i \omega \mu_{0} \sin \theta\left[A_{z}+\frac{1}{k^{2} r} \frac{\partial}{\partial r} A_{z}\right] \\
H_{\phi}=-\sin \theta \frac{\partial}{\partial r} A_{z} .
\end{gathered}
$$

The vector potential within the dielectric sphere may be obtained from a linear combination of solutions proportional to $e^{-i k_{1} r} / r$ and to $e^{i k_{1} \tau} / r$. The considerations are simplified if the secondary fields are finite at the origin. The vector potential within the dielectric sphere may be assumed to be of the form

$$
A_{z 1}=\frac{A^{\prime}}{r} e^{-i k_{1} r}+\frac{B^{\prime}}{r} \sin k_{1} r .
$$

The fields outside the sphere should represent an outgoing wave at large distances from the sphere. This suggests

$$
A_{z 2}=\frac{C^{\prime}}{r} e^{-i k_{2} \tau} \text {. }
$$

The constant $A^{\prime}$ characterizes the primary excitation and is related to the dipole moment $I h$ as

$$
A^{\prime}=\frac{I h}{4 \pi} \text {. }
$$

Continuity of $E_{\theta}$ and $H_{\phi}$ across the boundary of the sphere requires that the two conditions

$$
\begin{aligned}
A_{z 1}+\frac{1}{k_{1}^{2} r^{2}}\left[\frac{\partial}{\partial r}\left(r A_{z 1}\right)-A_{z 1}\right] & =A_{z 2}+\frac{1}{k_{2}^{2} r^{2}}\left[\frac{\partial}{\partial r}\left(r A_{z 2}\right)-A_{z 2}\right] \\
\frac{\partial}{\partial r}\left(r A_{z 1}\right)-A_{z 1} & =\frac{\partial}{\partial r}\left(r A_{z 2}\right)-A_{z 2}
\end{aligned}
$$

be satisfied for $r=a$. Substitution of (52) and (53) in $(55)$ and (56) results in

$$
\begin{array}{r}
A^{\prime}=\frac{C^{\prime} e^{-i y}}{2 i z}\left\{e ^ { i z } \left[\left(1+\frac{1}{i y}-\frac{1}{y^{2}}\right)(-1+i z)\right.\right. \\
\left.+(1+i y)\left(1-\frac{1}{i z}-\frac{1}{z^{2}}\right)\right]+e^{-i z} \\
\quad-(1+i y)\left(1+\frac{1}{i y}-\frac{1}{y^{2}}\right)(1+i z) \\
\left.\left.\left.z^{2}\right)\right]\right\}
\end{array}
$$

where $z$ and $y$ are defined by eqs (18) and (19). For spheres of a small radius, $y, z<<1$.

A series expansion of $e^{ \pm i z}$ results in

$$
A^{\prime} \approx C^{\prime} \frac{2 y^{2}+z^{2}}{3 y^{2}}
$$

which provides an agreement with (22). For spheres of large radii, $y, z<<1$. Only the terms of the square brackets, which are proportional to $y$ or $z$, are significant, and $A^{\prime}$ of (57) simplifies then to

$$
A^{\prime} \approx C^{\prime} e^{-i y}\left[\cos z+\frac{y}{z} i \sin z\right]
$$

which can be seen to agree with (31).

\subsection{Terminal Admittance of a Dielectric-Loaded, Wide-Angle Biconical Antenna}

The variational statement for the terminal admittance of a dielectric filled wide-angle biconical antenna is obtained following Tai [1949] as

$$
\begin{aligned}
Y_{t}=\frac{2 \pi 1}{\left[\int_{\theta_{0}}^{\pi-\theta_{0}} E_{\theta} d \theta\right]^{2}}\left\{\sqrt{\frac{\epsilon_{1}}{\mu_{0}}} \sum_{n} \frac{1}{n(n+1) N_{n} I_{n n}}\right. \\
\times\left[\int_{\theta_{0}}^{\pi-\theta_{0}} E_{\theta} I_{n}^{\prime}(\theta) \sin \theta d \theta\right]^{2} \\
-\sqrt{\frac{\epsilon_{2}}{\mu_{0}}} \sum_{k=1,3} \frac{1}{k(k+1) M_{k} I_{k k}} \\
\left.\times\left[\int_{\theta_{0}}^{\pi-\theta_{0}} E_{\theta} P_{k}^{\prime}(\theta) \sin \theta d \theta\right]^{2}\right\}
\end{aligned}
$$

where

$$
\begin{gathered}
I_{n n}=\int_{\theta_{0}}^{\pi-\theta_{0}} L_{n}^{2}(\theta) \sin \theta d \theta \\
I_{k k}=\int_{\theta}^{\pi} P_{k}^{2}(\theta) \sin \theta d \theta=\frac{2}{2 k+1} \\
N_{n}=\frac{S_{n}^{\prime}\left(k_{1} a\right)}{S_{n}\left(k_{1} a\right)} \\
M_{n}=\frac{R_{n}^{\prime}\left(k_{2} a\right)}{R_{n}\left(k_{2} a\right)} .
\end{gathered}
$$

$E_{\theta}$ is the aperture field at $r=a, \theta_{0}<\theta<\left(\pi-\theta_{0}\right)$ and $L_{n}(\theta)$ belong to a class of Legendre functions which vanish at $\theta=\theta_{0}, \pi / 2$ and $\pi-\theta_{0}$. The zero-order terminal admittance $Y_{t 0}$ is obtained with the aperture field approximation by (45), which may be expected to be sufficiently accurate for $\theta_{0}$ approaching $\frac{\pi}{2}$. It may be noted that

$$
\int_{\theta_{0}}^{\pi-\theta_{0}} E_{\theta} L_{n}^{\prime}(\theta) \sin \theta d \theta=-\left.A_{0} L_{n}(\theta)\right|_{\theta_{0}} ^{\pi-\theta_{0}}=0
$$




$$
\begin{gathered}
\int_{\theta_{0}}^{\pi-\theta_{0}} E_{\theta} P_{k}^{\prime}(\theta) \sin \theta d \theta=-2 A_{0} P_{k}(\cos \theta) \\
\int_{\theta_{0}}^{\pi-\theta_{0}} E_{\theta} d \theta=2 A_{0} \log \left(\tan \frac{\theta_{0}}{2}\right)
\end{gathered}
$$

Substitution of (65) to (67) in (66) can be seen to result in (46).

The calculation of the first-order terminal admittance $Y_{t 1}$ follows closely the analysis of Tai [1949]. Thus

$$
Y_{t 1}=Y_{t 0}-\frac{\alpha_{n}^{2}}{\sqrt{\frac{\epsilon_{1}}{\epsilon_{2}}} \beta_{n}+\gamma_{n n}}
$$

where

$$
\begin{aligned}
& \alpha_{n}=-\frac{i \sqrt{\mu_{0} / \epsilon_{2}}}{\pi K_{2}^{2}} \sum_{k=1,3, \ldots} \frac{(2 k+1) P_{k}\left(\cos \theta_{0}\right) I_{n k}}{2 M_{k}} \\
& \beta_{n}=\frac{i \sqrt{\mu_{0} / \epsilon_{2}}}{\pi K_{2}^{2}} \frac{n(n+1) I_{n n}}{2 N_{n}} \\
& \gamma_{n n}=-\frac{i \sqrt{\mu_{0} / \epsilon_{2}}}{\pi K_{2}^{2}} \sum_{k=1,3, \ldots} \frac{k(k+1)(2 k+1) I_{n k}^{2}}{4 M_{k}} \\
& I_{n k}=\frac{2 n P_{k}\left(\cos \theta_{0}\right) P_{n-1}\left(\cos \theta_{0}\right)}{\left(k^{2}+k-n^{2}-n\right)}, \quad(k \neq n) \\
& I_{n n}=\frac{4}{2 n+1}\left[\frac{P_{0}\left(\cos \theta_{0}\right) P_{1}\left(\cos \theta_{0}\right)}{1.3}\right. \\
& +\frac{P_{1}\left(\cos \theta_{0}\right) P_{2}\left(\cos \theta_{0}\right)}{3.5}+\ldots \\
& \left.+\frac{P_{n-2}\left(\cos \theta_{0}\right) P_{n-1}\left(\cos \theta_{0}\right)}{(2 n-3)(2 n-1)}\right] \\
& \cos \theta_{0} \approx \sqrt{\frac{21}{11+2 n(n+1)}}
\end{aligned}
$$

and $n$ is an odd integer.

After substituting the small argument approximations (42) and (41) for $N_{n}$ and $M_{k}$ in (70) and (69) the denominator of the correction term in (68) becomes

$$
\begin{aligned}
\sqrt{\frac{\epsilon_{1}}{\epsilon_{2}}} \beta_{n}+\gamma_{n n}=\frac{i \sqrt{\mu_{0} / \epsilon_{2}}}{\pi K_{2}^{2}} \frac{k_{2} a}{4}\left\{\frac{\epsilon_{1}}{\epsilon_{2}} \frac{n(n+1) I_{n n}}{2}\right. \\
\left.+\sum_{k=1,3, \ldots}(k+1)(2 k+1) I_{n k}^{2}-6 i\left(k_{2} a\right)^{3} I_{n 1}^{2}\right\} .
\end{aligned}
$$

Noting that $I_{n n}>0$, dielectric loading $\left(\epsilon_{1}>\epsilon_{2}\right)$ is seen to increase (75), which will result in a decreased difference between $Y_{t 1}$ and $Y_{t 0}$. The difference between $Y_{t 1}$ and $Y_{t 0}$ will therefore be even less than indicated by the numerical calculations of Tai [1949].

The limit of $\theta_{0} \rightarrow \frac{\pi}{2}$ may be examined quite readily. For $\theta_{0}$ approaching $\frac{\pi}{2}, n$ becomes large and

$$
\begin{gathered}
I_{n n} \approx \frac{1.27 \cos \theta_{0}}{2(2 n+1)} \\
I_{n k} \approx \frac{2 \sqrt{2} n P_{k}\left(\cos \theta_{0}\right)(-1)^{(n-1) / 2}}{\sqrt{\pi(n-1)}\left(k^{2}+k-n^{2}-n\right)} .
\end{gathered}
$$

For the odd integer $k>>1 I_{n k}$ may be further simplified to

$$
I_{n k} \approx \frac{4 n \sqrt{k+1} \cos \theta_{0}(-1)^{(n-1) / 2}(-1)^{(k-1) / 2}}{\pi \sqrt{n-1}\left(k^{2}+k-n^{2}-n\right)}
$$

Although strictly valid only for $k>>1$ and $\theta_{0} \rightarrow \pi / 2$, (78) exhibits an error of only 10 percent for $k$ as low as 1 . Substituting (76) in (70)

$$
\beta_{n} \approx-\frac{i}{\cos \theta_{0}} \sqrt{\frac{\epsilon_{2}}{\mu_{0}}}\left(k_{1} a\right) .
$$

The approximate behavior of (69) and (71) is most readily seen after substituting $I_{n k}$ of (78). Thus

$$
\begin{aligned}
& \alpha_{n}^{2} \approx-\frac{8 \pi \epsilon_{2}}{n \mu_{0}}\left(k_{2} a\right)^{2}\left[\sum_{k=1,3, \ldots} \frac{(2 k+1)(k+1)}{k\left(k^{2}+k-n^{2}-n\right)}\right]^{2} \\
& \gamma_{n n} \approx \frac{i 4 n^{2} k_{2} a}{(n-1) \pi} \sqrt{\frac{\epsilon_{2}}{\mu_{0}}} \sum_{k=1,3, \ldots} \frac{(k+1)^{2}(2 k+1)}{\left(n^{2}+n-k^{2}-k\right)^{2}} .
\end{aligned}
$$

The summations over $k$ of (80) and (81) are logarithmically infinite. Noting that $\cos \theta_{0} \sim n^{-1}$, $\alpha_{n n}^{2} \rightarrow 0$, while $\gamma_{n n} \rightarrow \infty$ as $\theta_{0} \rightarrow \frac{\pi}{2}$. Therefore $\left(Y_{t 1}-\right.$ $\left.Y_{t 0}\right) \rightarrow 0$ as $\theta_{0} \rightarrow \frac{\pi}{2}$, which is in agreement with the numerical calculations of Tai [1949] for $\epsilon_{2}=\epsilon_{1}$.

\section{References}

Keller, J. P., and Keller, M. B., A point dipole in spherically symmetric media, Math. Rev. 12, No. 3, 224 (March 1951). Also N.Y.U., Mathematics Research Group, Research Rep. No. EM-16 (1950).

King, R. W. P., Theory of Linear Antennas (Harvard Univ. Press, Cambridge, Mass., 1956).

Polk, C., Resonance and supergain effects in small ferromagnetically or dielectrically loaded biconical antennas, Proceed. Symposium on Electromagnetic Theory, The University of Toronto, Ontario, Canada, June 15-20, 1959 (Published as Special Supplement to the IRE Transactions on Antennas and Propagation, AP-y, S414-423, 1959).

Schelkunoff, S. A., Electromagnetic Waves (D. Van Nostrand Co., New York, 1943).

Sommerfeld A., Partial Differential Equations in Physics, 279-280 (Academic Press Inc., New York, 1949).

Stratton, J. A., Electromagnetic Theory, Sections 7.3 and 7.4 (McGraw-Hill Book Co., New York, 1941).

Tai, C. T. On the theory of biconical antennas, J. Appl. Phys. 19 No. 12, 1155-1160 (Dec. 1948).

Tai, C. T., Application of a variational principle to biconical antennas, J. Appl. Phys. 20 No. 11 1076-1084 (Nov. 1949).

Wait, J. R. The magnetic dipole antenna immersed in a conducting medium, Proc. IRE 40, No. 10, 1244-1245 (Oct. 1952).

Wheeler, H. A., Fundamental limitations of small antennas, Proc. IRE 35, No. 12, 1479-1484 (Dec. 1947).

(Paper 66D5-215) 